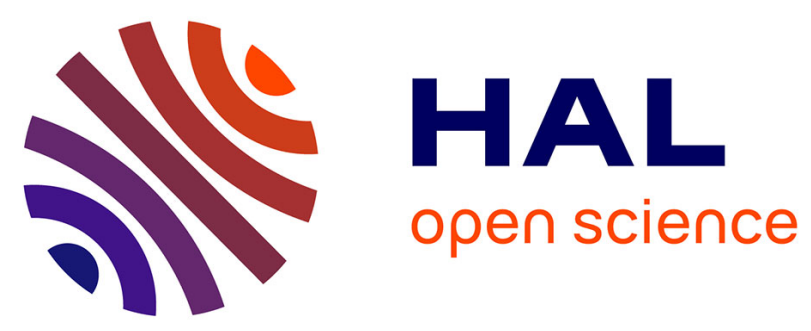

\title{
Stabilité d'un écoulement pulsé incompressible en tube cylindrique rigide
}

\author{
Ph. Dantan, F. de Jouvenel, C. Oddou
}

\section{To cite this version:}

Ph. Dantan, F. de Jouvenel, C. Oddou. Stabilité d'un écoulement pulsé incompressible en tube cylindrique rigide. Journal de Physique Lettres, 1976, 37 (10), pp.233-236. 10.1051/jphyslet:019760037010023300 . jpa-00231281

\section{HAL Id: jpa-00231281 https://hal.science/jpa-00231281}

Submitted on 1 Jan 1976

HAL is a multi-disciplinary open access archive for the deposit and dissemination of scientific research documents, whether they are published or not. The documents may come from teaching and research institutions in France or abroad, or from public or private research centers.
L'archive ouverte pluridisciplinaire HAL, est destinée au dépôt et à la diffusion de documents scientifiques de niveau recherche, publiés ou non, émanant des établissements d'enseignement et de recherche français ou étrangers, des laboratoires publics ou privés. 
Classification

Physics Abstracts

6.350

\title{
STABILITÉ D'UN ÉCOULEMENT PULSÉ INCOMPRESSIBLE EN TUBE CYLINDRIQUE RIGIDE
}

\author{
Ph. DANTAN, F. DE JOUVENEL et C. ODDOU \\ Université Paris-VII, L.B.H.P. Tour 33/34, 2, place Jussieu, 75221 Paris Cedex 05, France
}

(Reçu le 24 mai 1976, accepté le 8 juillet 1976)

\begin{abstract}
Résumé. - Des expériences de modélisation portant sur le déclenchement d'instabilités dans les écoulements sanguins artériels mettent en évidence lors de la transition une dépendance caractéristique entre les trois paramètres critiques que sont : le nombre de Reynolds, le paramètre de fréquence, et le paramètre d'amplitude. En généralisant, à partir des équations linéarisées de la dynamique des petites perturbations, la condition suffisante de stabilité des écoulements plans dépendant du temps au cas d'écoulements cylindriques, l'application de la condition trouvée permet d'interpréter de façon satisfaisante les résultats des expériences.
\end{abstract}

Abstract. - Model experiments concerning the onset of instabilities in arterial blood flows have shown characteristic relations between the three parameters : Reynolds number, frequency parameter and amplitude parameter. From linearized equations for the dynamics of small fluctuations, we extend the sufficiency condition for the stability of plane time dependent flows to the cylindrical geometry case. Theoretical results, thus obtained, are found to be in good agreement with experimental ones.

Le passage de l'état laminaire à l'état turbulent est actuellement un des phénomènes mal compris de la physique des fluides. Dans un écoulement permanent en géométrie cylindrique, le nombre de Reynolds $\overline{\mathrm{Re}}=2 \overline{R W} / v \overline{(W}$ vitesse débitante, $R$ rayon du tube, $v$ viscosité cinématique du fluide), rapport des effets d'inertie et de viscosité, est le paramètre fondamental caractéristique de l'état de mouvement des particules fluides. Ainsi, dans le cas d'un écoulement complètement développé, stationnaire, à l'intérieur d'un tube à parois lisses, de nombreux travaux expérimentaux après les études fondamentales de Reynolds [1] ont montré que la transition s'effectuait pour un nombre critique $\bar{R}_{c}$ de l'ordre de 2000 .

Cependant, dans le cas de mouvements instationnaires, peu d'études expérimentales ont été faites et l'analyse théorique de l'influence, sur la transition des paramètres de l'écoulement, s'avère plus complexe. Pourtant, cette étude est fondamentale dans le cas, par exemple, de la physiologie du système cardiovasculaire, où l'état du mouvement dans les gros conduits que constituent les artères, est très mal connu. Ici la transition, si elle existe, affecte les phénomènes hémodynamiques de propagation d'ondes, de génération de bruits audibles, de transport de constituants sanguins à travers les parois, etc...

Malheureusement, bien que ces écoulements soient associés pendant une partie du cycle cardiaque à des nombres de Reynolds instantanés dépassant large- ment le seuil critique établi en régime stationnaire, les considérations relatives à leur stabilité et l'apparition de turbulence dans ces conditions, n'avaient été, il y a quelques années, qu'à peine évoquées [2]. Récemment, afin d'étudier les mécanismes physiques qui interviennent dans le déclenchement des instabilités, des études expérimentales de modélisation d'écoulements pulsés en géométrie cylindrique, ont été effectuées [3, 4, 5]. Bien que les implications physiologiques de telles modélisations aient guidé leur conception, il est regrettable que les valeurs des paramètres de similitude :

(i) $\lambda=\tilde{W} / \bar{W}$ (paramètre d'amplitude) représentant le rapport du débit maximum $\pi R^{2} \tilde{W}$ correspondant à la composante harmonique, au débit de la composante continue $\pi R^{2} \bar{W}$.

(ii) $\alpha=R \sqrt{\omega / v}$ (paramètre de fréquence où $\omega$ désigne la pulsation de la composante harmonique) caractéristique des effets d'inertie (dus à la variation temporelle de la vitesse) et des effets de viscosité, soient très différentes de celles rencontrées dans les écoulements sanguins artériels.

Ainsi, dans de telles expériences, la composante pulsatile est généralement inférieure à la composante continue alors que dans les expériences in vivo on rencontre des valeurs du paramètre d'amplitude $\lambda$ supérieures à l'unité et pouvant aller jusqu'à la dizaine. De plus, ces expériences centrées sur l'étude 
de la croissance ou de l'amortissement d'une perturbation dans un écoulement de base sinusoïdalement accéléré ou décéléré font intervenir des fréquences telles que le paramètre de fréquence $\alpha$ est compris entre 1 et 10 , valeurs généralement plus faibles que dans les écoulements sanguins.

Pour expliquer les résultats de ces expériences de modélisation, il a été avancé l'hypothèse selon laquelle la génération de turbulence est due à l'amplification, dans la couche limite oscillante près des parois (zone à fort gradient généralement), des perturbations présentes à l'entrée du tube et dans le réservoir. Le postulat selon lequel les forces de viscosité sont supérieures aux forces d'inertie uniquement pendant une partie du cycle de l'écoulement pulsatile, conduit alors à la notion de nombre de Reynolds instantané [5], de temps de relaxation [3], et de nombre de Reynolds non stationnaire (cas du modèle quasi stationnaire utilisé pour déterminer la stabilité d'une couche limite plane soumise à un écoulement pulsatile [10]).

Par ailleurs, dans les conditions expérimentales où $\lambda>1$, certaines observations [6], en particulier l'apparition de perturbations dans le cœur de l'écoulement à l'instant où celui-ci s'inverse, conduisent à mettre en cause la forme du profil instantané des vitesses dans l'occurrence de la transition [7]. Cependant, ces explications théoriques consistent en l'assimilation de la distribution instantanée des vitesses locales à celle d'un écoulement stationnaire et la détermination, à partir de là, de la stabilité d'une petite perturbation superposée à l'écoulement de base considéré comme indépendant du temps.

Or, dans les expériences de modélisation citées précédemment, les écoulements de base considérés changent constamment dans le temps et de plus leur composante harmonique est faible devant leur composante stationnaire $(\lambda<1)$. Dans ces conditions la variation maximum du nombre de Reynolds instantané reste faible et par conséquent la dynamique de ces écoulements de base est dominée par les effets de viscosité prépondérants dans la composante continue. L'étude de leur stabilité abordée par ailleurs à l'aide de la théorie cinématique des ondes [11], doit donc être appréhendée par des nouvelles méthodes.

Pour cela, nous étendons l'analyse théorique linéarisée de Conrad et Criminale [8] au cas d'un écoulement en géométrie cylindrique axisymétrique d'axe $\mathrm{O} z$. A cet écoulement de base, de vitesse adimensionnée $U(x, t)$ (où $x=r / R$ ) dirigée suivant l'axe, est superposée une perturbation, supposée petite devant $U$, de composante radiale $v(x, t) \mathrm{e}^{i k z}$ et longitudinale $u(x, t) \mathrm{e}^{i k z}$. La fonction de courant $\psi(x, t)$ définie par:

$$
u=\frac{1}{x} \frac{\partial}{\partial x} x \psi, \quad v=-i k \psi
$$

étant introduite, les équations linéarisées de NavierStokes se réduisent alors à une équation d'OrrSommerfeld dépendant du temps :

$$
\begin{gathered}
\left(D-k^{2}\right) \psi=i k \operatorname{Re}\left[U\left(D-k^{2}\right) \psi-\psi\left(\frac{\partial U}{\partial x^{2}}-\frac{1}{x} \frac{\partial U}{\partial x}\right)\right]+\operatorname{Re} \frac{\partial}{\partial t}\left(D-k^{2}\right) \psi \\
D=\frac{\partial}{\partial x} \frac{1}{x} \frac{\partial}{\partial x} x .
\end{gathered}
$$

Par un certain nombre d'opérations analytiques (multiplication de (2) à gauche par $\psi^{*}$, complexe conjugué de $\psi$, addition de la relation résultante et de l'équation conjuguée de (2) multipliée par $\psi$, intégration sur la section en tenant compte des conditions aux limites de non-glissement et des conditions de symétrie, on obtient :

où

$$
2\left(I_{2}^{2}+2 k^{2} I_{1}^{2}+k^{4} I_{0}^{2}\right)=k \operatorname{Re} \int_{0}^{1} \frac{\partial U}{\partial x}\left[i \psi \frac{\partial}{\partial x}\left(x \psi^{*}\right)+(i \psi)^{*} \frac{\partial}{\partial x}(x \psi)\right] \mathrm{d} x-\operatorname{Re} \frac{\partial}{\partial t}\left(I_{1}^{2}+k^{2} I_{0}^{2}\right)
$$

$$
\begin{aligned}
& I_{0}^{2}=\int_{0}^{1} x|\psi|^{2} \mathrm{~d} x=\frac{1}{k^{2}} \int_{0}^{1}|v|^{2} x \mathrm{~d} x \\
& I_{1}^{2}=\int_{0}^{1}\left|\frac{\partial}{\partial x}(x \psi)\right|^{2} \mathrm{~d} x / x=\int_{0}^{1}|u|^{2} x \mathrm{~d} x \\
& I_{2}^{2}=\int_{0}^{1}|D \psi|^{2} x \mathrm{~d} x=\int_{0}^{1}\left|\frac{\partial U}{\partial x}\right|^{2} x \mathrm{~d} x .
\end{aligned}
$$

L'énergie adimensionnelle par unité de longueur d'onde transportée par la perturbation est :

$$
\varepsilon=\left(I_{1}^{2}+k^{2} I_{0}^{2}\right) / 2 .
$$


On désigne par $U_{M}^{\prime}$ la valeur maximum de $\partial U / \partial x$ au cours du temps et par $\left|U_{M}^{\prime}\right|_{\text {MAX }}$ le maximum de $\left|U_{M}^{\prime}\right|$ suivant $x$. Alors, par majoration de l'intégrale de l'équation (3), on tire :

$$
\operatorname{Re} \frac{\partial \varepsilon}{\partial t} \leqslant \operatorname{Re} k I_{0} I_{1}\left|U_{\mathrm{M}}^{\prime}\right|_{\mathrm{MAX}}-\left(I_{2}^{2}+2 k^{2} I_{1}^{2}+k^{4} I_{0}^{2}\right) .
$$

Il en résulte ainsi une condition suffisante pour que l'énergie cinétique $\varepsilon$ des perturbations soit décroissante (condition nécessaire de stabilité) :

$$
\operatorname{Re} \leqslant \frac{A}{\left|U_{\mathrm{M}}^{\prime}\right|_{\mathrm{MAX}}} ; \quad A=\left(I_{2}^{2}+2 k^{2} I_{1}^{2}+k^{4} I_{0}^{2}\right) / k I_{0} I_{1} .
$$

Cette condition est appliquée au cas de l'écoulement pulsé développé rencontré dans les expériences de modélisation précédemment mentionnées. Le profil des vitesses axiales de l'écoulement de base $U(x, t)$ est donné par l'expression analytique [9] :

$$
\begin{aligned}
& \frac{W}{\bar{W}}=2\left(1-x^{2}\right)+\frac{\lambda}{C(\alpha)}[B(x, \alpha) \sin \omega t+(1-A(x, \alpha) \cos \omega t)] \\
& A(x, \alpha)=(\operatorname{ber}(\alpha) \text { ber }(\alpha x)+\operatorname{bei}(\alpha) \text { bei }(\alpha x)) /\left(\operatorname{ber}^{2}(\alpha)+\operatorname{bei}^{2}(\alpha)\right) \\
& B(x, \alpha)=(\operatorname{bei}(\alpha) \operatorname{ber}(\alpha x)-\operatorname{ber}(\alpha) \operatorname{bei}(\alpha x)) /\left(\operatorname{ber}^{2}(\alpha)+\operatorname{bei}^{2}(\alpha)\right) \\
& C^{2}(\alpha)=(1-2 D / \alpha)^{2}+(2 E / \alpha)^{2} \\
& D \quad=-\left.\frac{\mathrm{d} B}{\mathrm{~d}(\alpha x)}\right|_{x=1}, \quad E=\left.\frac{\mathrm{d} A}{\mathrm{~d}(\alpha x)}\right|_{x=1}
\end{aligned}
$$

où ber et bei sont les fonctions de Kelvin d'ordre 0 . Pour cet écoulement, le gradient de vitesse normalisé maximum dans le temps est égal à :

$$
\frac{W_{\mathrm{M}}^{\prime}}{\bar{W}}=-4 x+\frac{\lambda}{C(\alpha)} \sqrt{(\mathrm{d} B / \mathrm{d} x)^{2}+(\mathrm{d} A / \mathrm{d} x)^{2}} .
$$

Dans sa variation en fonction de $x,\left|W_{M}^{\prime}\right| / \bar{W}$ présente un maximum qui dépend de $\lambda$ et $\alpha$ (Fig. 1) qu'on désigne par $\left|W_{\mathrm{M}}^{\prime}\right|_{\mathrm{MAX}} / \bar{W}$. Pour différentes valeurs de $\alpha$ la relation (5) est tracée. Les courbes

$$
\operatorname{Re}_{\mathrm{c}}=A /\left|W_{\mathrm{M}}^{\prime}\right|_{\text {MAX }} / \bar{W}
$$

ainsi obtenues en fonction de $\lambda$ présentent un pic comme on peut s'en rendre compte sur la figure 2

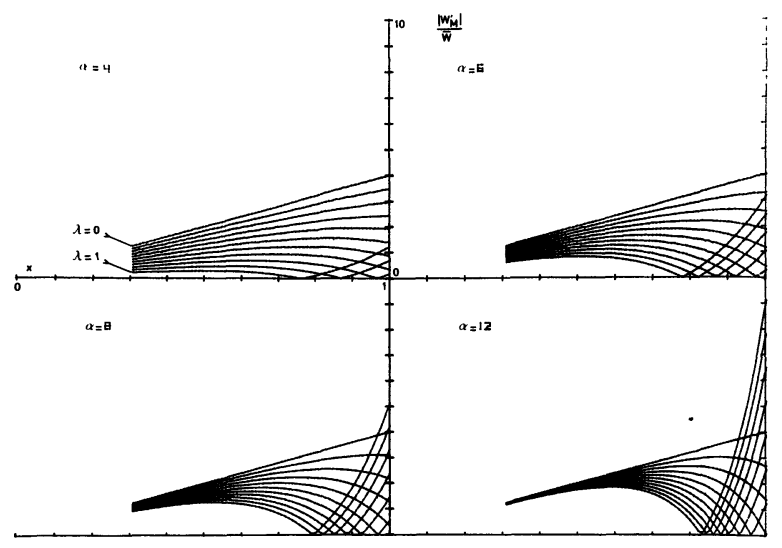

FIG. 1. - Variation radiale du gradient de vitesse normalisé dans un écoulement pulsé pour différentes valeurs des paramètres de fréquence $\alpha$ et d'amplitude $\lambda$.

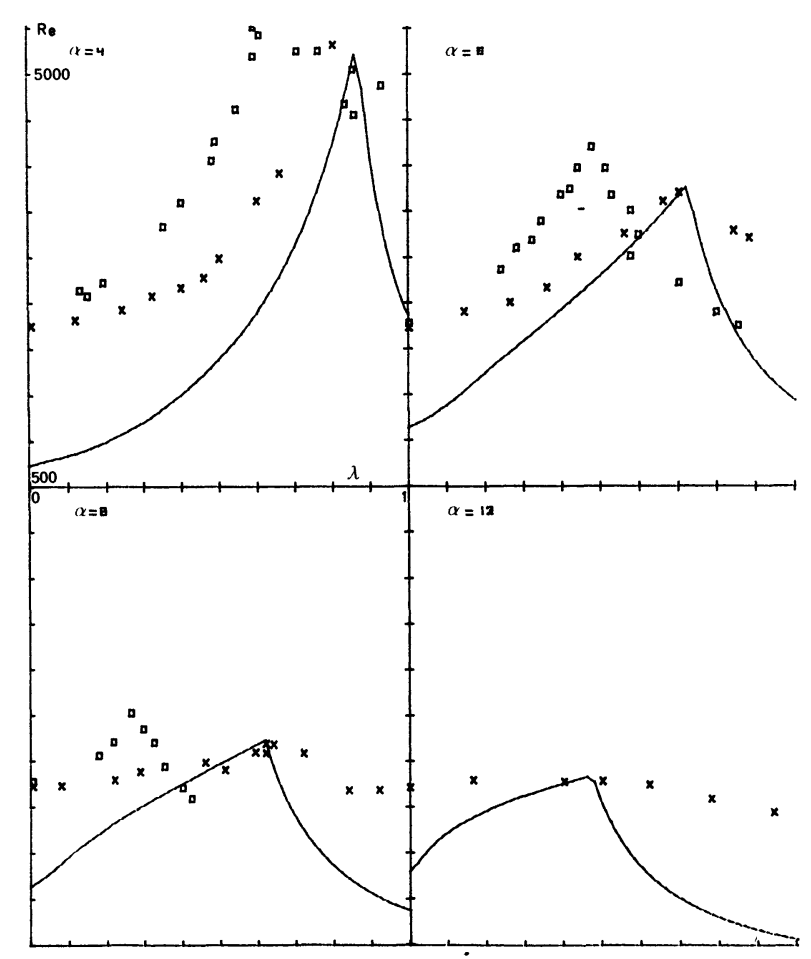

FIG. 2. - Comparaison des résultats théoriques et expérimentaux : variation théorique en fonction du paramètre d'amplitude $\lambda$ du nombre de Reynolds moyen critique pour différentes valeurs du paramètre de fréquence $\alpha$. $\square \square \square$ Expériences de Sarpkaya [4]. $x \times \times$ Expériences de Gilbrecht et Combs [5].

et une variation d'allure générale identique aux résultats expérimentaux : en particulier, et indépendamment de la valeur de $A$, l'abscisse $\lambda$ pour laquelle 
les courbes présentent un pic coïncide bien avec les résultats expérimentaux. D'autre part, le paramètre $A$ qui reste indéterminé tant que la perturbation n'est pas connue a été ajusté afin de faire coïncider les maxima théoriques et expérimentaux du nombre de Reynolds critique, et ce, pour chaque valeur de $\alpha$.

On constate alors que les points expérimentaux de Gilbrecht et Combs [5] se répartissent au-dessus de la courbe théorique (cf. Fig. 2), ce qui est cohérent puisque la relation utilisée est une condition suffisante de stabilité marginale, alors que les observations portent sur des perturbations d'amplitude finie. De plus, il est à noter le fait que les maxima théoriques et expérimentaux de $\operatorname{Re}_{\mathrm{c}}$ apparaissent pour des valeurs de $\lambda$ très voisines. Les résultats expérimentaux de Sarpkaya [4], tout en ayant une variation semblable se décalent légèrement des courbes théoriques. Ceci est certainement dû au fait que, d'une part, l'écoulement utilisé par cet auteur n'est pas purement sinusoïdal et, d'autre part, ses valeurs de $\alpha$ sont un peu différentes.
Une autre source d'écart entre les résultats théoriques et expérimentaux est certainement due au paramètre théorique $A$ dont l'expression est très complexe. Ce facteur qui est caractéristique de la perturbation doit dépendre implicitement de l'écoulement de base, en particulier des paramètres $\lambda$ et $\alpha$, mais cette théorie ne suffit pas à rendre compte de cette dépendance puisqu'elle ne permet pas le calcul explicite du champ des vitesses perturbées. A ce stade préliminaire de la théorie, on a supposé que $A$ ne dépendait pas de $\lambda$. De plus, sa dépendance avec $\alpha$ n'a pu être prise en compte qu'empiriquement. On peut noter cependant un bon accord qualitatif entre les résultats théoriques et les profils très particuliers des variations du nombre de Reynolds critique en fonction de $\alpha$ et $\lambda$ déterminés expérimentalement. Néanmoins, la connaissance théorique des perturbations conduirait à un meilleur accord quantitatif (détermination de $A$ ). Un calcul numérique de résolution de l'équation (2) est en cours qui devrait permettre d'atteindre cet objectif.

\section{Bibliographie}

[1] ReYNOLdS, Phil. Trans. 174 (1883) 935-982.

[2] MC DonalD, D. A., In Blood flow in arteries (2nd Ed) 71, Ed. Arnold, London (1974).

[3] Yellin, E. L., Circ. Res. 19 (1966) 791.

[4] Sarpkaya, T., Trans. of the A.S.M.E., Series D, Journal of basic Engineering 88 (1966) 589.

[5] Gilbrecht, D. A., Combs, G. D., In Developments in Theoretical and Applied Mechanics (Plenum Press, Inc, New York), Vol. 1 (1963) 292.
[6] Sergeev, S. I., Fluid Dyn. 1 (1966) 121.

[7] Dantan, Ph., De Jouvenel, F., Oddou, C., J. Physique Lett. 37 (1976) L-157.

[8] Conrad, P. W., Criminale, W. O., Z. Angew. Math. Phys. 16 (1965a) 233-254.

[9] Uchida, S., Z. Angew. Math. Phys. 7 (1956) 403.

[10] OBREMSKI et MORKOvin, AIAA Journal 7 (1969) 1298.

[11] LANDHAL, J. Fluid Mech. 56 (1972) 775. 